\title{
Optimality conditions for fractional variational problems with Caputo-Fabrizio fractional derivatives
}

\author{
Jianke Zhang*, Xiaojue Ma and Lifeng Li
}

"Correspondence: jiankezh@163.com

Department of Mathematics,

School of Science, Xi'an University

of Posts and Telecommunications,

Chang'an Road, Xi'an, China

\begin{abstract}
In this paper, we study the necessary and sufficient optimality conditions for problems of the fractional calculus of variations with a Lagrange function depending on a Caputo-Fabrizio fractional derivative. The new kernel of Caputo-Fabrizio fractional derivative has no singularity, which is critical to interpreting the memory aftermath of the system. This property was not precisely illustrated in the previous definitions. Two special cases of fractional variational problems are considered to demonstrate the application of the optimality conditions.
\end{abstract}

Keywords: fractional variational problems; optimality conditions; Caputo-Fabrizio fractional derivative

\section{Introduction}

Since the introduction of fractional calculus of variations by Riewe [1], fractional calculus has played an important role in dealing with many natural dynamical processes. Riewe showed that the traditional Lagrangian and Hamiltonian mechanics cannot be used with nonconservative forces such as friction. However, fractional calculus may be better to describe the behavior of the natural processes because of its memory property. So, considerable progress has been made to determine necessary and sufficient conditions that any extremal for the variational functional with fractional calculus must satisfy in recent years. Agrawal [2-4] studied the fractional Euler-Lagrange equations for general fractional variational problems (FVP) involving Riemann-Liouville, Caputo and Riesz fractional derivatives. Almeida investigated optimality conditions for fractional variational problems with a Lagrangian depending on the Riesz-Caputo derivative [5] and the Caputo-Katugampola derivative [6]. In [7], Almeida exhibited the conditions of optimality for functionals depending on Caputo fractional integrals and derivatives, on indefinite integrals and on the presence of time delay. Xu and Agrawal [8] deduced the Euler-Lagrange equation of the fractional variational problem involving a modified Hilfer fractional derivative. Farhadinia [9], Fard [10] and Soolaki [11] established the necessary optimality conditions for fuzzy fractional variational problems by using the generalized Hukuhara differentiability concept.

The most popular fractional calculi are Riemann-Liouville (RL) and Caputo type. But it is well known that the two derivatives have some drawbacks. For example, the RL deriva-

(c) The Author(s) 2017. This article is distributed under the terms of the Creative Commons Attribution 4.0 International License (http://creativecommons.org/licenses/by/4.0/), which permits unrestricted use, distribution, and reproduction in any medium, provided you give appropriate credit to the original author(s) and the source, provide a link to the Creative Commons license, and indicate if changes were made. 
tive of a constant is not zero and it demands initial conditions of non-integer order which are not physically determined, and the Caputo derivative requires higher conditions of regularity for differentiability, which is specified only for differentiable functions.

In 2015, Caputo and Fabrizio [12] proposed a new fractional derivative with nonsingular kernel which conveniently portrays the performance of material heterogeneities and the structures with different scales. The main difference between the Caputo derivative and the Caputo-Fabrizio (CF) fractional derivative is that the new kernel has no singularity. Losada and Nieto studied some properties of the new fractional derivative [13], and several researchers tried to utilize it for solving fractional differential equations (see [14] and the references therein). Kumar et al. [15] studied a time-fractional modified Kawahara equation with a CF fractional derivative. In [16], Singh et al. analyzed the El Nino-Southern Oscillation model in the global climate with the CF fractional derivative and obtained the solution by using the iterative method. By using the CF fractional derivative, Hristov [1719] expressed the Cattaneo constitutive equation with Jeffrey's fading memory naturally resulting in a heat conduction equation with a relaxation term, and this approach allowed to see the physical background of the CF time fractional derivative and demonstrate how other constitutive equations could be modified with non-singular fading memories.

Fractional derivatives of variable order were also used to set up the mathematical models for engineering practice, especially in the fields of heat [20] and fluid flows [21, 22]. Zaky and Machado [23] derived the generalized necessary conditions for the fractional optimal control problems and proposed an efficient numerical scheme. Bhrawy and Zaky studied the accurate numerical schemes for the problems of variable-order fractional Schrodinger equations [24]. In [25], the Nabla Euler-Lagrange equations of the discrete fractional variational problems were given. Garra et al. [26] proposed the necessary conditions for the fractional Herglotz variational problems with generalized Caputo derivatives. Tavares et al. [27] studied the necessary conditions for the constrained FVP of variable order. However, it is difficult to solve the fractional differential equations. Some novel numerical techniques [28-33] were proposed to solve the class of problems. In [29-31], Kumar et al. introduced a new numerical algorithm, which was named q-homotopy analysis transform algorithm, to obtain the approximate solutions for the fractional model of regularized long-wave equation, the nonlinear fractional dynamical model and the time-fractional Rosenau-Hyman equation.

However, some issues were pointed out against both derivatives, including the one in Caputo sense and the one in Riemann-Liouville sense. As Sheikh [22] pointed out, the $\mathrm{CF}$ fractional derivative as the kernel in integral was non-singular but was still nonlocal. Some researchers also concluded that the operator was not a derivative with fractional order but a filter with fractional parameter. The fractional parameter can then be viewed as a filter regulator. To overcome the above drawbacks, Yang et al. [34] proposed a new fractional derivative involving the normalized sinc function without singular kernel. Atangana and Baleanu introduced a new operator with fractional order based upon the generalized Mittag-Leffler function [35]. Their operators have all the benefits of that of the CF derivative in addition to the kernel being nonlocal and non-singular. The non-locality of the kernel gives better description of the memory within the structure with different scale.

The main aim of this paper is to present the optimality conditions for fractional variational problems involving the $\mathrm{CF}$ fractional derivative. This paper is structured as follows. In Section 2, the basic definitions and notations are introduced, including the CF frac- 
tional derivatives. In Sections 3 and 4, the optimality conditions for fractional variational problems are derived. In Section 5, the optimality condition for the fractional Herglotz problem is proposed. Finally, an example and conclusion are proposed in Sections 6 and 7 , respectively.

\section{Preliminaries}

In this section, we recall some basic concepts with regard to the Caputo fractional derivative [36] and the CF fractional derivative [12]. Given a function $x(t):[a, b] \rightarrow \mathbf{R}$, the Caputo fractional derivative of $x$ of order $\alpha \in(0,1)$ is defined as

$$
{ }^{C} D_{a+}^{\alpha} x(t)=\frac{1}{\Gamma(1-\alpha)} \frac{d}{d t} \int_{a}^{t} \frac{1}{(t-\tau)^{\alpha}}[x(\tau)-x(a)] d \tau .
$$

If $x$ is of class $C^{1}$, then

$$
{ }^{C} D_{a+}^{\alpha} x(t)=\frac{1}{\Gamma(1-\alpha)} \int_{a}^{t} \frac{1}{(t-\tau)^{\alpha}} x^{\prime}(\tau) d \tau .
$$

The new CF fractional derivative $[12,13]$ can be obtained by changing the kernel $(t-\tau)^{-\alpha}$ into the function $\exp (-\alpha(t-\tau) /(1-\alpha))$ and $1 / \Gamma(1-\alpha)$ into $M(\alpha) /(1-\alpha)$. That is,

$$
{ }^{C F} D_{a+}^{\alpha} x(t)=\frac{M(\alpha)}{1-\alpha} \int_{a}^{t} \exp \left(-\frac{\alpha(t-\tau)}{1-\alpha}\right) x^{\prime}(\tau) d \tau
$$

where $M(\alpha)$ is a normalization function such that $M(0)=M(1)=1$. It is clear that if $x$ is a constant function, then ${ }^{C F} D^{\alpha} x(t)=0$ as in the usual Caputo derivative, but contrary to the usual Caputo derivative, the kernel does not have singularity for $t=\tau$.

Definition 1 ([12]) Let $x \in H^{1}(a, b), b>a, \alpha \in(0,1)$, then the CF fractional derivative is described as (1), where $M(\alpha)$ stands for a normalization function such that $M(0)=$ $M(1)=1$. If the function does not belong to $x \in H^{1}(a, b)$, the derivative can be reconstructed as

$$
{ }^{C F} D_{a+}^{\alpha} x(t)=\frac{\alpha M(\alpha)}{1-\alpha} \int_{a}^{t} \exp \left(-\frac{\alpha(t-\tau)}{1-\alpha}\right)(x(t)-x(\tau)) d \tau .
$$

Remark 1 The kernel function of the CF fractional derivative is an exponential function. As we introduced in Section 1, there are several non-singular kernel functions such as the normalized sinc function [34], the generalized Mittag-Leffler function [35], Meijer Gfunction [37] and Fox H-function [38], which can be used to define the fractional derivative and integral. So there is a problem, and what kind of kernel function is better? Some researchers [20, 22, 39-41] compared the actual effects of CF derivatives with AtanganaBaleanu derivatives in the following practical problems.

(1) For the generalized Casson fluid model with heat generation and chemical reaction, Sheikh $[22,40]$ pointed out that, for a unit time, the velocities obtained via Atangana-Baleanu and CF derivatives are identical. Velocities for the time less than 1 show little variation and for time bigger than 1 this variation increases. In [39], the Atangana-Baleanu and the CF derivatives were used to extend the model of reaction-diffusion known as Allen-Cahn model, and the modified models were both 
solved numerically and numerical simulations presented for different values of alpha.

(2) Koca [41] pointed out that the CF derivative is a filter not a fractional derivative based upon the fact that the kernel used is local and may not be able to portray more accurately the complex system via which the flow of heat is taking place.

In summary, the existing studies for some practical problems have not consistently shown that the Atangana-Baleanu or the CF derivative is more effective. For fractional variational problems, which class of the fractional derivative is more effective? Further research is needed on the basis of practical fractional variational problems.

We denote an auxiliary fractional integral and a differential as

$$
I_{b-}^{\alpha} x(t):=\frac{M(1-\alpha)}{\alpha} \int_{t}^{b} x(\tau) \exp \left(-\frac{(1-\alpha)(\tau-t)}{\alpha}\right) d \tau,
$$

which is not the CF fractional integral as in [13], and

$$
{ }^{C F} D_{b-}^{\alpha} x(t):=\frac{M(\alpha)}{1-\alpha} \frac{d}{d t} \int_{t}^{b} x(\tau) \exp \left(-\frac{\alpha(\tau-t)}{1-\alpha}\right) d \tau .
$$

Proposition 1 Let $x$ be a continuous function and $y$ be of class $C^{1}$. Then

$$
\int_{a}^{b} x(t)^{C F} D_{a+}^{\alpha} y(t) d t=\left[y(t) I_{b-}^{1-\alpha} x(t)\right]_{t=a}^{t=b}-\int_{a}^{b} y(t)^{C F} D_{b-}^{\alpha} x(t) d t .
$$

Proof From the definition of CF fractional derivative, we have

$$
\int_{a}^{b} x(t)^{C F} D_{a+}^{\alpha} y(t) d t=\frac{M(\alpha)}{1-\alpha} \int_{a}^{b} \int_{a}^{t} x(t) \exp \left(-\frac{\alpha(t-\tau)}{1-\alpha}\right) y^{\prime}(\tau) d \tau d t .
$$

By Dirichlet's formula and integrating by parts, we get

$$
\begin{aligned}
& \frac{M(\alpha)}{1-\alpha} \int_{a}^{b} \int_{a}^{t} x(t) \exp \left(-\frac{\alpha(t-\tau)}{1-\alpha}\right) y^{\prime}(\tau) d \tau d t \\
& =\frac{M(\alpha)}{1-\alpha} \int_{a}^{b} \int_{t}^{b} x(\tau) \exp \left(-\frac{\alpha(\tau-t)}{1-\alpha}\right) y^{\prime}(t) d \tau d t \\
& =\left[y(t) \cdot \frac{M(\alpha)}{1-\alpha} \int_{t}^{b} x(\tau) \exp \left(-\frac{\alpha(\tau-t)}{1-\alpha}\right) d \tau\right]_{t=a}^{t=b} \\
& \quad-\int_{a}^{b} y(t) \cdot \frac{M(\alpha)}{1-\alpha} \frac{d}{d t} \int_{t}^{b} x(\tau) \exp \left(-\frac{\alpha(\tau-t)}{1-\alpha}\right) d \tau d t \\
& =\left[y(t) I_{b-}^{1-\alpha} x(t)\right]_{t=a}^{t=b}-\int_{a}^{b} y(t)^{C F} D_{b-x}^{\alpha} x(t) d t .
\end{aligned}
$$

\section{Optimality conditions for FVP}

In this section, we consider the following problem with a $\mathrm{CF}$ fractional derivative. Given $x \in C^{1}[a, b]$,

$$
\min J(x)=\int_{a}^{b} L\left(t, x(t),{ }^{C F} D_{a+}^{\alpha} x(t)\right) d t,
$$


with $x(a)=x_{a}$ and $x(b)=x_{b}$, where $x_{a}, x_{b} \in \mathbf{R}$. The assumptions are as follows:

1. $L:[a, b] \times \mathbf{R}^{2} \rightarrow \mathbf{R}$ is continuously differentiable with respect to the second and third arguments;

2. Given any $x$, the map $t \mapsto{ }^{C F} D_{b-}^{\alpha}\left(\partial_{3} L\left(t, x(t),{ }^{C F} D_{a+}^{\alpha} x(t)\right)\right)$ is continuous.

Hereafter, we denote $\partial_{i} f\left(x_{1}, \ldots, x_{n}\right):=\frac{\partial f}{\partial x_{i}}\left(x_{1}, \ldots, x_{n}\right)$ for a function $f: S \subseteq \mathbf{R}^{n} \rightarrow \mathbf{R}$. We propose the Euler-Lagrange equation of (2). At the solutions of (2), the first variation of the functional must be vanished.

Theorem 1 Let $x$ be a solution of (2). Then $x$ is a solution of the fractional Euler-Lagrange equation for all $t \in[a, b]$

$$
\partial_{2} L\left(t, x(t),{ }^{C F} D_{a+}^{\alpha} x(t)\right)-{ }^{C F} D_{b-}^{\alpha}\left(\partial_{3} L\left(t, x(t),{ }^{C F} D_{a+}^{\alpha} x(t)\right)\right)=0 .
$$

Proof Consider $x+\epsilon h$ to be a variation of $x$, and $h:[a, b] \rightarrow \mathbf{R}$ is a function of class $C^{1}[a, b]$ such that the boundary conditions of $h(a)=h(b)=0$ hold. Let $j(\epsilon)=J(x+\epsilon h)$, since $x$ is a solution of (2), then $j^{\prime}(0)=0$.

Computing $\left.j^{\prime}(\epsilon)\right|_{\epsilon=0}$ and using Proposition 1, we have

$$
\begin{aligned}
\int_{a}^{b} & \partial_{2} L\left(t, x(t),{ }^{C F} D_{a+}^{\alpha} x(t)\right) h(t) d t+\int_{a}^{b} \partial_{3} L\left(t, x(t),{ }^{C F} D_{a+}^{\alpha} x(t)\right){ }^{C F} D_{a+}^{\alpha} h(t) d t \\
= & \int_{a}^{b}\left[\partial_{2} L\left(t, x(t),{ }^{C F} D_{a+}^{\alpha} x(t)\right)-{ }^{C F} D_{b-}^{\alpha}\left(\partial_{3} L\left(t, x(t),{ }^{C F} D_{a+}^{\alpha} x(t)\right)\right)\right] h(t) d t \\
& +\left[h(t) I_{b-}^{1-\alpha}\left(\partial_{3} L\left(t, x(t),{ }^{C F} D_{a+}^{\alpha} x(t)\right)\right)\right]_{t=a}^{t=b} \\
= & 0 .
\end{aligned}
$$

From the boundary conditions of $h(a)=h(b)=0$ and $h$ is arbitrary elsewhere, we get

$$
\partial_{2} L\left(t, x(t),{ }^{C F} D_{a+}^{\alpha} x(t)\right)-{ }^{C F} D_{b-}^{\alpha}\left(\partial_{3} L\left(t, x(t),{ }^{C F} D_{a+}^{\alpha} x(t)\right)\right)=0, \quad \forall t \in[a, b] .
$$

Definition 2 A function $x$ that is a solution of (3) is called an extremal for $J$.

Remark 2 The Euler-Lagrange equation (3) is easily extended to the case of several variations.

Definition 3 We say that $L(t, x, y)$ is convex in $K \subseteq \mathbf{R}^{3}$ if $\partial_{2} L$ and $\partial_{3} L$ exist and are continuous, and the condition

$$
L\left(t, x+x_{1}, y+y_{1}\right)-L(t, x, y) \geq \partial_{2} L(t, x, y) x_{1}+\partial_{3} L(t, x, y) y_{1}
$$

holds for every $(t, x, y),\left(t, x+x_{1}, y+y_{1}\right) \in K$.

Theorem 2 If the function $L$ as in (2) is convex in $[a, b] \times \mathbf{R}^{2}$, then each solution of the fractional Euler-Lagrange equation (3) minimizes J, when restricted to the boundary conditions of $x(a)=x_{a}$ and $x(b)=x_{b}$. 
Proof Let $x$ be a solution of (3) and $x+\epsilon h$ be a variation of $x$ with $|\epsilon| \ll 1$, and $h \in C^{1}[a, b]$ with $h(a)=h(b)=0$. Using Proposition 1, we get

$$
\begin{aligned}
J(x+\epsilon h)-J(x) \\
=\int_{a}^{b}\left[L\left(t, x(t)+\epsilon h(t),{ }^{C F} D_{a+}^{\alpha} x(t)+\epsilon{ }^{C F} D_{a+}^{\alpha} h(t)\right)-L\left(t, x(t),{ }^{C F} D_{a+}^{\alpha} x(t)\right)\right] d t \\
\geq \int_{a}^{b}\left[\partial_{2} L\left(t, x(t),{ }^{C F} D_{a+}^{\alpha} x(t)\right) \epsilon h(t)+\partial_{3} L\left(t, x(t),{ }^{C F} D_{a+}^{\alpha} x(t)\right) \epsilon{ }^{C F} D_{a+}^{\alpha} h(t)\right] d t \\
=\int_{a}^{b} \partial_{2} L\left(t, x(t),{ }^{C F} D_{a+}^{\alpha} x(t)\right) \epsilon h(t) d t+\epsilon\left[h(t) I_{b-}^{1-\alpha} \partial_{3} L\left(t, x(t),{ }^{C F} D_{a+}^{\alpha} x(t)\right)\right]_{t=a}^{t=b} \\
\quad-\int_{a}^{b} \epsilon h(t){ }^{C F} D_{b-}^{\alpha} \partial_{3} L\left(t, x(t),{ }^{C F} D_{a+}^{\alpha} x(t)\right) d t \\
=\int_{a}^{b}\left[\partial_{2} L\left(t, x(t),{ }^{C F} D_{a+}^{\alpha} x(t)\right)-{ }^{C F} D_{b-}^{\alpha} \partial_{3} L\left(t, x(t),{ }^{C F} D_{a+}^{\alpha} x(t)\right)\right] \epsilon h(t) d t .
\end{aligned}
$$

Since $x$ is a solution of (3), then

$$
\partial_{2} L\left(t, x(t),{ }^{C F} D_{a+}^{\alpha} x(t)\right)-{ }^{C F} D_{b-}^{\alpha} \partial_{3} L\left(t, x(t),{ }^{C F} D_{a_{+}}^{\alpha} x(t)\right)=0 .
$$

Thus, $J(x+\epsilon h) \geq J(x), x$ is a local minimizer of $J$.

Next, we consider a more general class of fractional variational problems for $A \in(a, b)$ and the functional

$$
\min J(x)=\int_{A}^{b} L\left(t, x(t),{ }^{C F} D_{a+}^{\alpha} x(t)\right) d t,
$$

with $x(t) \in C^{1}[a, b]$ and $x(a)=x_{a}, x(b)=x_{b}$, where $x_{a}, x_{b} \in \mathbf{R}$. The assumptions are as previous ones for $L\left(t, x(t),{ }^{C F} D_{a+}^{\alpha} x(t)\right)$.

Theorem 3 If $x$ is a solution of (4), then $x$ satisfies

$$
{ }^{C F} D_{A-}^{\alpha}\left(\partial_{3} L\left(t, x(t),{ }^{C F} D_{a+}^{\alpha} x(t)\right)\right)-{ }^{C F} D_{b-}^{\alpha}\left(\partial_{3} L\left(t, x(t),{ }^{C F} D_{a+}^{\alpha} x(t)\right)\right)=0
$$

on $[a, A]$,

$$
\partial_{2} L\left(t, x(t),{ }^{C F} D_{a+}^{\alpha} x(t)\right)-{ }^{C F} D_{b-}^{\alpha}\left(\partial_{3} L\left(t, x(t),{ }^{C F} D_{a+}^{\alpha} x(t)\right)\right)=0
$$

on $[A, b]$ and

$$
I_{A-}^{1-\alpha}\left(\partial_{3} L\left(t, x(t),{ }^{C F} D_{a+}^{\alpha} x(t)\right)\right)-I_{b-}^{1-\alpha}\left(\partial_{3} L\left(t, x(t),{ }^{C F} D_{a+}^{\alpha} x(t)\right)\right)=0
$$

at $t=a$.

Proof Let $x$ be a solution of (4) and $x+\epsilon h$ be a variation of $x$ with $|\epsilon| \ll 1$, and $h \in C^{1}[a, b]$ with $h(A)=h(b)=0$. Let $j(\epsilon)=J(x+\epsilon h)$, since $x$ is a solution of $(4)$, then $j^{\prime}(0)=0$. 
Computing $\left.j^{\prime}(\epsilon)\right|_{\epsilon=0}$ and using Proposition 1, we have

$$
\begin{aligned}
& \int_{A}^{b} \partial_{2} L\left(t, x(t),{ }^{C F} D_{a+}^{\alpha} x(t)\right) h(t) d t+\int_{A}^{b} \partial_{3} L\left(t, x(t),{ }^{C F} D_{a+}^{\alpha} x(t)\right)^{C F} D_{a+}^{\alpha} h(t) d t \\
&=\int_{a}^{b}\left[\partial_{2} L\left(t, x(t),{ }^{C F} D_{a+}^{\alpha} x(t)\right) h(t)+\partial_{3} L\left(t, x(t),{ }^{C F} D_{a+}^{\alpha} x(t)\right)^{C F} D_{a+}^{\alpha} h(t)\right] d t \\
& \quad-\int_{a}^{A}\left[\partial_{2} L\left(t, x(t),{ }^{C F} D_{a+}^{\alpha} x(t)\right) h(t)+\partial_{3} L\left(t, x(t),{ }^{C F} D_{a+}^{\alpha} x(t)\right)^{C F} D_{a+}^{\alpha} h(t)\right] d t \\
&=\int_{A}^{b} \partial_{2} L\left(t, x(t),{ }^{C F} D_{a+}^{\alpha} x(t)\right) h(t) d t+\int_{a}^{b} \partial_{3} L\left(t, x(t),{ }^{C F} D_{a+}^{\alpha} x(t)\right)^{C F} D_{a+}^{\alpha} h(t) d t \\
& \quad-\int_{a}^{A} \partial_{3} L\left(t, x(t),{ }^{C F} D_{a+}^{\alpha} x(t)\right)^{C F} D_{a+}^{\alpha} h(t) d t \\
&=\int_{A}^{b} \partial_{2} L\left(t, x(t),{ }^{C F} D_{a+}^{\alpha} x(t)\right) h(t) d t+\left[h(t) I_{b-}^{1-\alpha}\left(\partial_{3} L\left(t, x(t),{ }^{C F} D_{a+}^{\alpha} x(t)\right)\right)\right]_{t=a}^{t=b} \\
&-\int_{a}^{b} h\left(t{ }^{C F} D_{b-}^{\alpha}\left(\partial_{3} L\left(t, x(t),{ }^{C F} D_{a+}^{\alpha} x(t)\right)\right) d t\right. \\
&-\left[h(t) I_{A-}^{1-\alpha}\left(\partial_{3} L\left(t, x(t),{ }^{C F} D_{a+}^{\alpha} x(t)\right)\right)\right]_{t=a}^{t=A} \\
&+\int_{a}^{A} h(t){ }^{C F} D_{A-}^{\alpha}\left(\partial_{3} L\left(t, x(t),{ }^{C F} D_{a+}^{\alpha} x(t)\right)\right) d t \\
&= 0 .
\end{aligned}
$$

From $h(A)=h(b)=0$, the above equation deduces the following:

$$
\begin{array}{rl}
\int_{a}^{A} & h(t)\left[{ }^{C F} D_{A-}^{\alpha}\left(\partial_{3} L\left(t, x(t),{ }^{C F} D_{a+}^{\alpha} x(t)\right)\right)-{ }^{C F} D_{b_{-}}^{\alpha}\left(\partial_{3} L\left(t, x(t),{ }^{C F} D_{a+}^{\alpha} x(t)\right)\right)\right] d t \\
& +\int_{A}^{b} h(t)\left[\partial_{2} L\left(t, x(t),{ }^{C F} D_{a+}^{\alpha} x(t)\right)-{ }^{C F} D_{b_{-}}^{\alpha}\left(\partial_{3} L\left(t, x(t),{ }^{C F} D_{a+}^{\alpha} x(t)\right)\right)\right] d t \\
& +\left.h(a)\left[I_{A-}^{1-\alpha}\left(\partial_{3} L\left(t, x(t),{ }^{C F} D_{a+}^{\alpha} x(t)\right)\right)-I_{b_{-}}^{1-\alpha}\left(\partial_{3} L\left(t, x(t),{ }^{C F} D_{a+}^{\alpha} x(t)\right)\right)\right]\right|_{t=a} \\
= & 0 .
\end{array}
$$

Since $h$ is arbitrary elsewhere, we get the three necessary conditions.

\section{The fractional variational problem with holonomic constraint}

Let $\left(x_{1}(t), x_{2}(t)\right) \in C^{1}[a, b] \times C^{1}[a, b]$ and $x_{a}^{1}, x_{a}^{2}, x_{b}^{1}, x_{b}^{2} \in \mathbf{R}$ be fixed such that $\left(x_{1}(a), x_{2}(a)\right)=$ $\left(x_{a}^{1}, x_{a}^{2}\right)$ and $\left(x_{1}(b), x_{2}(b)\right)=\left(x_{b}^{1}, x_{b}^{2}\right)$. Consider the following problem:

$\min J\left(x_{1}, x_{2}\right)=\int_{a}^{b} L\left(t, x_{1}(t), x_{2}(t),{ }^{C F} D_{a+}^{\alpha} x_{1}(t),{ }^{C F} D_{a+}^{\alpha} x_{2}(t)\right) d t$

such that $g\left(t, x_{1}(t), x_{2}(t)\right)=0$.

Assume that the following conditions hold:

1. $L:[a, b] \times \mathbf{R}^{4} \rightarrow \mathbf{R}$ is continuously differentiable with respect to its $i$ th arguments for $i=2,3,4,5$; 
2. Given any $\mathbf{x}=\left(x_{1}, x_{2}\right)$, the map $t \mapsto{ }^{C F} D_{b-}^{\alpha}\left(\partial_{i} L\left(t, x_{1}(t), x_{2}(t),{ }^{C F} D_{a_{+}}^{\alpha} x_{1}(t),{ }^{C F} D_{a+}^{\alpha} x_{2}(t)\right)\right)$ is continuous for $i=4,5$;

3. The admissible function $g:[a, b] \times \mathbf{R}^{2}$ is continuously differentiable with respect to its $i$ th arguments for $i=2,3$.

Next, we denote that

$$
\mathbf{x}=\left(x_{1}(t), x_{2}(t)\right) \text { and }{ }^{C F} D_{a+}^{\alpha} \mathbf{x}=\left({ }^{C F} D_{a+}^{\alpha} x_{1}(t),{ }^{C F} D_{a+}^{\alpha} x_{2}(t)\right) .
$$

Theorem 4 Let the function $x$ be a solution of (5)-(6). If $\partial_{3} g(t, \mathbf{x}) \neq 0$ for all $t \in[a, b]$, then there is a continuous function $\lambda(t):[a, b] \rightarrow \mathbf{R}$ such that $x$ is a solution of the fractional differential equation

$$
\partial_{2} L\left(t, \mathbf{x},{ }^{C F} D_{a+}^{\alpha} \mathbf{x}\right)-{ }^{C F} D_{b_{-}}^{\alpha}\left(\partial_{4} L\left(t, \mathbf{x},{ }^{C F} D_{a+}^{\alpha} \mathbf{x}\right)\right)+\lambda(t) \partial_{2} g(t, \mathbf{x})=0
$$

and

$$
\partial_{3} L\left(t, \mathbf{x},{ }^{C F} D_{a+}^{\alpha} \mathbf{x}\right)-{ }^{C F} D_{b-}^{\alpha}\left(\partial_{5} L\left(t, \mathbf{x},{ }^{C F} D_{a+}^{\alpha} \mathbf{x}\right)\right)+\lambda(t) \partial_{3} g(t, \mathbf{x})=0
$$

on $[a, b]$.

Proof Let $\mathbf{x}$ be a solution of (5)-(6) and $\mathbf{x}+\epsilon \mathbf{h}$ be a variation of $\mathbf{x}$ with $|\epsilon| \ll 1$, and $\mathbf{h}=\left(h_{1}(t), h_{2}(t)\right) \in C^{1}[a, b] \times C^{1}[a, b]$ with $\mathbf{h}(a)=\mathbf{h}(b)=(0,0)$. From the assumption of $\partial_{3} g(t, \mathbf{x}) \neq 0$ for all $t \in[a, b]$ and the implicit function theorem, there exists a unique function $h_{2}\left(\epsilon, h_{1}\right)$ such that $\left(x_{1}+\epsilon h_{1}, x_{2}+\epsilon h_{2}\right)$ satisfies (6). So, we have the following equation satisfied for all $t \in[a, b]$ :

$$
g\left(t, x_{1}(t)+\epsilon h_{1}(t), x_{2}(t)+\epsilon h_{2}(t)\right)=0
$$

Then

$$
\left.\frac{\partial g\left(t, x_{1}(t)+\epsilon h_{1}(t), x_{2}(t)+\epsilon h_{2}(t)\right)}{\partial \epsilon}\right|_{\epsilon=0}=0,
$$

that is,

$$
\partial_{2} g(t, \mathbf{x}) h_{1}(t)+\partial_{3} g(t, \mathbf{x}) h_{2}(t)=0 .
$$

Since $\partial_{3} g(t, \mathbf{x}) \neq 0$ for all $t \in[a, b]$, we denote

$$
\lambda(t)=-\frac{\partial_{3} L\left(t, \mathbf{x},{ }^{C F} D_{a+}^{\alpha} \mathbf{x}\right)-{ }^{C F} D_{b-}^{\alpha}\left(\partial_{5} L\left(t, \mathbf{x},{ }^{C F} D_{a_{+}}^{\alpha} \mathbf{x}\right)\right)}{\partial_{3} g(t, \mathbf{x})} .
$$

From (10) and (11), we get

$$
\begin{aligned}
\lambda(t) \partial_{2} g(t, \mathbf{x}) h_{1}(t)= & {\left[\partial_{3} L\left(t, \mathbf{x},{ }^{C F} D_{a+}^{\alpha} \mathbf{x}\right)\right.} \\
& \left.-{ }^{C F} D_{b-}^{\alpha}\left(\partial_{5} L\left(t, \mathbf{x},{ }^{C F} D_{a+}^{\alpha} \mathbf{x}\right)\right)\right] h_{2}(t) .
\end{aligned}
$$


Let $j(\epsilon)=J(\mathbf{x}+\epsilon \mathbf{h})$, since $\mathbf{x}$ is a solution of (5), the first variation of $J$ must vanish, then $j^{\prime}(0)=0$. Computing $\left.j^{\prime}(\epsilon)\right|_{\epsilon=0}$, we have

$$
\begin{aligned}
& \int_{a}^{b}\left[\partial_{2} L\left(t, \mathbf{x},{ }^{C F} D_{a+}^{\alpha} \mathbf{x}\right) h_{1}(t)+\partial_{3} L\left(t, \mathbf{x},{ }^{C F} D_{a+}^{\alpha} \mathbf{x}\right) h_{2}(t)\right. \\
& \quad+\partial_{4} L\left(t, \mathbf{x},{ }^{C F} D_{a+}^{\alpha} \mathbf{x}\right)^{C F} D_{a+}^{\alpha} h_{1}(t) \\
& \left.\quad+\partial_{5} L\left(t, \mathbf{x},{ }^{C F} D_{a+}^{\alpha} \mathbf{x}\right){ }^{C F} D_{a+}^{\alpha} h_{2}(t)\right] d t=0 .
\end{aligned}
$$

Using Proposition 1 and $\mathbf{h}(a)=\mathbf{h}(b)=(0,0)$, we obtain

$$
\begin{aligned}
& \int_{a}^{b}\left\{\left[\partial_{2} L\left(t, \mathbf{x},{ }^{C F} D_{a+}^{\alpha} \mathbf{x}\right)-{ }^{C F} D_{b-}^{\alpha}\left(\partial_{4} L\left(t, \mathbf{x},{ }^{C F} D_{a+}^{\alpha} \mathbf{x}\right)\right)\right] h_{1}(t)\right. \\
& \left.\quad+\left[\partial_{3} L\left(t, \mathbf{x},{ }^{C F} D_{a+}^{\alpha} \mathbf{x}\right)-{ }^{C F} D_{b-}^{\alpha}\left(\partial_{5} L\left(t, \mathbf{x},{ }^{C F} D_{a+}^{\alpha} \mathbf{x}\right)\right)\right] h_{2}(t)\right\} d t=0 .
\end{aligned}
$$

Inserting (12)-(13) into the above equation, we obtain

$$
\int_{a}^{b}\left[\partial_{2} L\left(t, \mathbf{x},{ }^{C F} D_{a+}^{\alpha} \mathbf{x}\right)-{ }^{C F} D_{b-}^{\alpha}\left(\partial_{4} L\left(t, \mathbf{x},{ }^{C F} D_{a+}^{\alpha} \mathbf{x}\right)\right)+\lambda(t) \partial_{2} g(t, \mathbf{x})\right] h_{1}(t) d t=0 .
$$

Since $h_{1}$ is arbitrary elsewhere, we get the necessary condition as follows:

$$
\partial_{2} L\left(t, \mathbf{x},{ }^{C F} D_{a+}^{\alpha} \mathbf{x}\right)-{ }^{C F} D_{b-}^{\alpha}\left(\partial_{4} L\left(t, \mathbf{x},{ }^{C F} D_{a+}^{\alpha} \mathbf{x}\right)\right)+\lambda(t) \partial_{2} g(t, \mathbf{x})=0 .
$$

From (11), we have another necessary condition

$$
\partial_{3} L\left(t, \mathbf{x},{ }^{C F} D_{a+}^{\alpha} \mathbf{x}\right)-{ }^{C F} D_{b-}^{\alpha}\left(\partial_{5} L\left(t, \mathbf{x},{ }^{C F} D_{a+}^{\alpha} \mathbf{x}\right)\right)+\lambda(t) \partial_{3} g(t, \mathbf{x})=0 .
$$

Theorem 5 Let the function $L\left(t, \mathbf{x},{ }^{C F} D_{a+}^{\alpha} \mathbf{x}\right)$ as in (5) be convex in $[a, b] \times \mathbf{R}^{4}, g:[a, b] \times$ $\mathbf{R}^{2} \rightarrow \mathbf{R}$ is continuously differentiable with respect to its ith arguments for $i=2,3$. For the continuous function $\lambda(t):[a, b] \rightarrow \mathbf{R}$ be given in (11) and $\partial_{3} g(t, \mathbf{x}) \neq 0$ for all $t \in[a, b]$, if $\mathbf{x}$ is a solution of (7) subject to (6), then $\mathbf{x}$ is also a solution of (5)-(6).

Proof Since $\mathbf{x}$ is a solution of (7) subject to (6) and $g:[a, b] \times \mathbf{R}^{2} \rightarrow \mathbf{R}$ is continuously differentiable with respect to its $i$ th arguments for $i=2,3$, then $\mathbf{x}$ satisfies (10). From $\partial_{3} g(t, \mathbf{x}) \neq 0$ for all $t \in[a, b]$, we get

$$
h_{2}(t)=\frac{-\partial_{2} g(t, \mathbf{x}) h_{1}(t)}{\partial_{3} g(t, \mathbf{x})} .
$$

On the other hand, if $\mathbf{x}+\epsilon \mathbf{h}$ is a variation of $\mathbf{x}$, we have

$$
\begin{aligned}
J(\mathbf{x}+\epsilon \mathbf{h})-J(\mathbf{x})= & \int_{a}^{b}\left[L\left(t, \mathbf{x}+\epsilon \mathbf{h},{ }^{C F} D_{a+}^{\alpha}(\mathbf{x}+\epsilon \mathbf{h})\right)-L\left(t, \mathbf{x},{ }^{C F} D_{a_{+}}^{\alpha} \mathbf{x}\right)\right] d t \\
\geq & \int_{a}^{b}\left[\partial_{2} L\left(t, \mathbf{x},{ }^{C F} D_{a+}^{\alpha} \mathbf{x}\right) \epsilon h_{1}(t)+\partial_{3} L\left(t, \mathbf{x},{ }^{C F} D_{a+}^{\alpha} \mathbf{x}\right) \epsilon h_{2}(t)\right. \\
& \left.+\partial_{4} L\left(t, \mathbf{x},{ }^{C F} D_{a+}^{\alpha} \mathbf{x}\right) \epsilon{ }^{C F} D_{a+}^{\alpha} h_{1}(t)+\partial_{5} L\left(t, \mathbf{x},{ }^{C F} D_{a+}^{\alpha} \mathbf{x}\right) \epsilon{ }^{C F} D_{a+}^{\alpha} h_{2}(t)\right] d t .
\end{aligned}
$$


Integrating by parts for the right-hand side of the above inequation, using Proposition 1 and $\mathbf{h}(a)=\mathbf{h}(b)=(0,0)$, we obtain

$$
\begin{aligned}
J(\mathbf{x}+\epsilon \mathbf{h})-J(\mathbf{x}) \geq & \int_{a}^{b}\left\{\left[\partial_{2} L\left(t, \mathbf{x},{ }^{C F} D_{a+}^{\alpha} \mathbf{x}\right)-{ }^{C F} D_{b-}^{\alpha}\left(\partial_{4} L\left(t, \mathbf{x},{ }^{C F} D_{a+}^{\alpha} \mathbf{x}\right)\right)\right] \epsilon h_{1}(t)\right. \\
& \left.+\left[\partial_{3} L\left(t, \mathbf{x},{ }^{C F} D_{a+}^{\alpha} \mathbf{x}\right)-{ }^{C F} D_{b-}^{\alpha}\left(\partial_{5} L\left(t, \mathbf{x},{ }^{C F} D_{a+}^{\alpha} \mathbf{x}\right)\right)\right] \epsilon h_{2}(t)\right\} d t .
\end{aligned}
$$

Using (14) and since $\mathbf{x}$ is a solution of (7), we get

$$
\begin{aligned}
J(\mathbf{x}+\epsilon \mathbf{h})-J(\mathbf{x}) \geq & \int_{a}^{b}\left[\partial_{2} L\left(t, \mathbf{x},{ }^{C F} D_{a+}^{\alpha} \mathbf{x}\right)-{ }^{C F} D_{b-}^{\alpha}\left(\partial_{4} L\left(t, \mathbf{x},{ }^{C F} D_{a+}^{\alpha} \mathbf{x}\right)\right)\right. \\
& \left.+\lambda(t) \partial_{2} g(t, \mathbf{x})\right] \epsilon h_{1}(t) d t=0 .
\end{aligned}
$$

Thus, $J(\mathbf{x}+\epsilon \mathbf{h}) \geq J(\mathbf{x}), \mathbf{x}$ is a solution of $J$.

\section{The fractional Herglotz problem}

The fractional Herglotz problem is to determine a curve $x \in C^{1}[a, b]$ subject to $x(a)=x_{a}$ and $x(b)=x_{b}$ such that $z$ is the solution of the following system:

$$
\begin{aligned}
& z^{\prime}(t)=L\left(t, x(t),{ }^{C F} D_{a+}^{\alpha} x(t), z(t)\right), \quad t \in[a, b] \\
& z(a)=z_{a}
\end{aligned}
$$

and $\left.z(t)\right|_{t=b}$ is a minimum. This problem was studied in [6] based on the CaputoKatugampola fractional derivative. However, contrary to the Caputo-Fabrizio derivative in this paper, the Caputo-Katugampola fractional derivative has singularity for $t=\tau$. For any function $x(t)$, the map $t \mapsto{ }^{C F} D_{a+}^{\alpha} x(t)$ is continuously differentiable and the map $t \mapsto{ }^{C F} D_{b-}^{\alpha}\left(\lambda(t) \partial_{3} L\left(t, x(t),{ }^{C F} D_{a+}^{\alpha} x(t), z(t)\right)\right)$ is continuous with

$$
\lambda(t)=\exp \left(-\int_{a}^{t} \partial_{4} L\left(\tau, x(\tau),{ }^{C F} D_{a+}^{\alpha} x(\tau), z(\tau)\right) d \tau\right) .
$$

It can be known that the solution $z$ depends on $t$ and $x$. If we consider the function of $h(t) \in C^{1}[a, b]$ with $h(a)=h(b)=0$ and any sufficiently small real number $\epsilon$, then $x+$ $\epsilon h \in C^{1}[a, b]$ lies in the neighborhood $N_{\epsilon}(x)$. We substitute $x$ by $x+\epsilon h$, the solution $z$ also depends on $\epsilon$, and it is also differentiable with respect to $\epsilon$.

Theorem 6 Let the function $x$ be such that $z(b)$ as in (15)-(16) attains a minimum. Then $x$ is a solution of the fractional differential equation

$$
\lambda(t) \partial_{2} L\left(t, x(t),{ }^{C F} D_{a+}^{\alpha} x(t), z(t)\right)-{ }^{C F} D_{b-}^{\alpha}\left(\lambda(t) \partial_{3} L\left(t, x(t),{ }^{C F} D_{a+}^{\alpha} x(t), z(t)\right)\right)=0 .
$$

Proof Let the function of $h(t) \in C^{1}[a, b]$ with $h(a)=h(b)=0$ and any sufficiently small real number $\epsilon$. Using $x+\epsilon h$ is a variation of $x$ and the solution $z$ is given by

$$
\phi(t)=\left.\frac{d}{d \epsilon} z(t, x+\epsilon h)\right|_{\epsilon=0} .
$$


From (15) and (18), we get

$$
\phi^{\prime}(t)=\left.\frac{d}{d t} \frac{d}{d \epsilon} z(t, x+\epsilon h)\right|_{\epsilon=0}
$$

and

$$
\frac{d}{d t} z(t, x(t)+\epsilon h(t))=L\left[t, x(t)+\epsilon h(t),{ }^{C F} D_{a+}^{\alpha} x(t)+\epsilon^{C F} D_{a+}^{\alpha} h(t), z(t, x(t)+\epsilon h(t))\right]
$$

Thus, we have

$$
\begin{aligned}
\phi^{\prime}(t)= & \left.\frac{d}{d \epsilon} L\left[t, x(t)+\epsilon h(t),{ }^{C F} D_{a+}^{\alpha} x(t)+\epsilon^{C F} D_{a+}^{\alpha} h(t), z(t, x(t)+\epsilon h(t))\right]\right|_{\epsilon=0} \\
= & \partial_{2} L\left(t, x(t),{ }^{C F} D_{a+}^{\alpha} x(t), z(t)\right) h(t)+\partial_{3} L\left(t, x(t),{ }^{C F} D_{a+}^{\alpha} x(t), z(t)\right){ }^{C F} D_{a+}^{\alpha} h(t) \\
& +\partial_{4} L\left(t, x(t),{ }^{C F} D_{a+}^{\alpha} x(t), z(t)\right) \phi(t) .
\end{aligned}
$$

We denote that

$$
a(t)=\partial_{4} L\left(t, x(t),{ }^{C F} D_{a+}^{\alpha} x(t), z(t)\right)
$$

and

$$
b(t)=\partial_{2} L\left(t, x(t),{ }^{C F} D_{a+}^{\alpha} x(t), z(t)\right) h(t)+\partial_{3} L\left(t, x(t),{ }^{C F} D_{a+}^{\alpha} x(t), z(t)\right)^{C F} D_{a+}^{\alpha} h(t) .
$$

Then the above differential equation can be deduced as

$$
\phi^{\prime}(t)=a(t) \phi(t)+b(t)
$$

which is

$$
\left[\phi^{\prime}(t)-a(t) \phi(t)\right] \lambda(t)=b(t) \lambda(t)
$$

then

$$
\begin{aligned}
& \int_{a}^{t} d(\phi(\tau) \lambda(\tau))=\int_{a}^{t} b(\tau) \lambda(\tau) d \tau \\
& \Rightarrow \phi(t) \lambda(t)-\phi(a)= \int_{a}^{t} \lambda(\tau)\left[\partial_{2} L\left(\tau, x(\tau),{ }^{C F} D_{a+}^{\alpha} x(\tau), z(\tau)\right) h(\tau)\right. \\
&\left.+\partial_{3} L\left(\tau, x(\tau),{ }^{C F} D_{a+}^{\alpha} x(\tau), z(\tau)\right){ }^{C F} D_{a+}^{\alpha} h(\tau)\right] d \tau \\
&= \int_{a}^{t} \lambda(\tau) \partial_{2} L\left(\tau, x(\tau),{ }^{C F} D_{a+}^{\alpha} x(\tau), z(\tau)\right) h(\tau) d \tau \\
&+\left.\left\{h(\tau) I_{b-}^{1-\alpha}\left[\lambda(\tau) \partial_{3} L\left(\tau, x(\tau),{ }^{C F} D_{a+}^{\alpha} x(\tau), z(\tau)\right)\right]\right\}\right|_{\tau=a} ^{\tau=t} \\
&-\int_{a}^{t} h(\tau)^{C F} D_{b-}^{\alpha}\left[\lambda(\tau) \partial_{3} L\left(\tau, x(\tau),{ }^{C F} D_{a+}^{\alpha} x(\tau), z(\tau)\right)\right] d \tau .
\end{aligned}
$$


Replacing $t$ by $b$ in the above equation, since $z(a)$ is fixed and $z(b)$ is the minimum, we have $\phi(a)=\phi(b)=0$. By $h(a)=h(b)=0$ and the arbitrariness of $h$ in $(a, b)$, we get

$$
\lambda(t) \partial_{2} L\left(t, x(t),{ }^{C F} D_{a+}^{\alpha} x(t), z(t)\right)-{ }^{C F} D_{b-}^{\alpha}\left(\lambda(t) \partial_{3} L\left(t, x(t),{ }^{C F} D_{a+}^{\alpha} x(t), z(t)\right)\right)=0
$$

for all $t \in[a, b]$.

\section{Example}

Example Let us consider the following unconstrained fractional variational problem for $0<\alpha<1$ :

$$
\min J(x)=\int_{0}^{1}\left({ }^{C F} D_{0+}^{\alpha} x(t)+f(t)\right)^{2} d t
$$

such that $\quad x(0)=0, \quad x(1)=1$,

where $f(t)=\frac{M(\alpha)}{\alpha}\left[e^{-\frac{\alpha t}{1-\alpha}}-1\right]$ and $M(\alpha)=1+\sin (\alpha \pi)$.

For this problem, according to the Euler-Lagrange equation as in (3), we get

$$
{ }^{C F} D_{1-}^{\alpha}\left[{ }^{C F} D_{0+}^{\alpha} x(t)+f(t)\right]=0 .
$$

By direct substitution, it can be shown that $x(t)=t$ is the unique solution to this problem. In fact, for the case of $x(t)=t$, we have

$$
\begin{aligned}
{ }^{C F} D_{0_{+}}^{\alpha} x(t) & =\frac{M(\alpha)}{1-\alpha} \int_{0}^{t} x^{\prime}(\tau) \exp \left(-\frac{\alpha(t-\tau)}{1-\alpha}\right) d \tau \\
& =\frac{M(\alpha)}{1-\alpha} \cdot \frac{1-\alpha}{\alpha} \int_{0}^{t} d\left[\exp \left(-\frac{\alpha(t-\tau)}{1-\alpha}\right)\right] \\
& =\left.\frac{M(\alpha)}{\alpha} \exp \left(-\frac{\alpha(t-\tau)}{1-\alpha}\right)\right|_{\tau=0} ^{\tau=t} \\
& =\frac{M(\alpha)}{\alpha}\left[1-e^{-\frac{\alpha t}{1-\alpha}}\right] .
\end{aligned}
$$

It can be observed that as $\alpha \rightarrow 1$, the fractional variational problem becomes

$$
\begin{aligned}
& \min J(x)=\int_{0}^{1}\left(x^{\prime}(t)+f(t)\right)^{2} d t \\
& \text { such that } x(0)=0, \quad x(1)=1,
\end{aligned}
$$

where $f(t)=-1$ since $M(1)=1 . x(t)=t$ is obviously the unique solution to this problem.

\section{Conclusions}

In this paper, we have discussed the necessary and sufficient optimality conditions for problems of the fractional calculus of variations with a Lagrange function depending on a Caputo-Fabrizio fractional derivative. The advantage of the new fractional derivative has no singularity, which was not precisely illustrated in the previous definitions. Two 
classes of FVP are considered to demonstrate the application of the optimality conditions. However, the Euler-Lagrange equations for FVP are in general difficult to solve. As the future works, we should develop numerical methods to solve this problem.

\section{Acknowledgements}

The author would like to express his gratitude to the anonymous reviewers for their very valuable remarks and comments. This work is supported by the National Natural Science Foundation of China (Grant No. 11701446, 11601420, 11401469, 60974082) and the Science Plan Foundation of the Education Bureau of Shaanxi Province (No. 2013JK 1130).

\section{Competing interests}

The authors declare that they have no competing interests.

\section{Authors' contributions}

All authors jointly worked on the results and they read and approved the final manuscript.

\section{Publisher's Note}

Springer Nature remains neutral with regard to jurisdictional claims in published maps and institutional affiliations.

Received: 9 April 2017 Accepted: 2 October 2017 Published online: 09 November 2017

\section{References}

1. Riewe, F: Nonconservative Lagrangian and Hamiltonian mechanics. Phys. Rev. E 53, 1890-1899 (1996)

2. Agrawal, OP: Formulation of Euler-Lagrange equations for fractional variational problems. J. Math. Anal. Appl. 272 , 368-379 (2002)

3. Agrawal, OP: Fractional variational calculus and the transversality conditions. J. Phys. A 39, 10375-10384 (2006)

4. Agrawal, OP: Fractional variational calculus in terms of Riesz fractional derivatives. J. Phys. A 40, 6287-6303 (2007)

5. Almeida, R: Fractional variational problems with the Riesz-Caputo derivative. Appl. Math. Lett. 25, 142-148 (2012)

6. Almeida, R: Variational problems involving a Caputo-type fractional derivative. J. Optim. Theory Appl. 174(1), 276-294 (2017) doi:10.1007/s10957-016-0883-4

7. Almeida, R: Fractional variational problems depending on indefinite integrals and with delay. Bull. Malays. Math. Soc. 39, 1515-1528 (2016)

8. Xu, Y, Agrawal, OP: New fractional operators and application to fractional variational problem. Comput. Math. Appl. (2016). doi:10.1016/j.camwa.2016.04.008

9. Farhadinia, B: Necessary optimality conditions for fuzzy variational problems. Inf. Sci. 181(7), 1348-1357 (2011)

10. Fard, OS, Salehi, M: A survey on fuzzy fractional variational problems. J. Comput. Appl. Math. 271, 71-82 (2014)

11. Soolaki, J, Fard, OS, Borzabadi, AH: Generalized Euler-Lagrange equations for fuzzy variational problems. SeMA J. 73 131-148 (2016)

12. Caputo, M, Fabrizio, M: A new definition of fractional derivative without singular kernel. Prog. Fract. Differ. Appl. 1 , 73-85 (2015)

13. Losada, J, Nieto, JJ: Properties of a new fractional derivative without singular kernel. Prog. Fract. Differ. Appl. 1, 87-92 (2015)

14. Baleanu, D, Mousalou, A, Rezapour, S: A new method for investigating approximate solutions of some fractional integro-differential equations involving the Caputo-Fabrizio derivative. Adv. Differ. Equ. 2017, 51 (2017) doi:10.1186/s13662-017-1088-3

15. Kumar, D, Singh, J, Baleanu, D: Modified Kawahara equation within a fractional derivative with non-singular kernel. Therm. Sci. (2017). doi:10.2298/TSCI160826008K

16. Singh, J, Kumar, D, Nieto, JJ: Analysis of an El Nino-Southern Oscillation model with a new fractional derivative. Chaos Solitons Fractals 99, 109-115 (2017)

17. Hristov, J: Transient heat diffusion with a non-singular fading memory: from the Cattaneo constitutive equation with Jeffrey's kernel to the Caputo-Fabrizio time-fractional derivative. Therm. Sci. 20(2), 757-762 (2016)

18. Hristov, J: Steady-state heat conduction in a medium with spatial non-singular fading memory: derivation of Caputo-Fabrizio space-fractional derivative with Jeffrey's kernel and analytical solutions. Therm. Sci. 21, 827-839 (2017)

19. Hristov, J: Frontiers in Fractional Calculus: Chapter 10, pp. 235-295. Bentham Science Publishers, Sharjah (2017)

20. Yang, XJ: Fractional derivatives of constant and variable orders applied to anomalous relaxation models in heat transfer problems. Therm. Sci. 21, 1161-1171 (2017)

21. Yang, XJ, Machado, JAT: A new fractional operator of variable order: application in the description of anomalous diffusion. Phys. A, Stat. Mech. Appl. 481, 276-283 (2017)

22. Sheikh, NA, Ali, F, Saqib, M, et al.: Comparison and analysis of the Atangana-Baleanu and Caputo-Fabrizio fractional derivatives for generalized Casson fluid model with heat generation and chemical reaction. Results Phys. 7, 789-800 (2017)

23. Zaky, MA, Machado, JAT: On the formulation and numerical simulation of distributed-order fractional optimal control problems. Commun. Nonlinear Sci. Numer. Simul. 52, 177-189 (2017)

24. Bhrawy, AH, Zaky, MA: Highly accurate numerical schemes for multi-dimensional space variable-order fractional Schrodinger equations. Comput. Math. Appl. 73(6), 1100-1117 (2017)

25. Abdeljawad, T: Nabla Euler-Lagrange equations in discrete fractional variational calculus within Riemann and Caputo. International Journal of Mathematics and Computation (2017) arXiv:1703.06751 [math.CA]

26. Garra, R, Taverna, GS, Torres, DFM: Fractional herglotz variational principles with generalized Caputo derivatives. Chaos Solitons Fractals (2017). doi:10.1016/j.chaos.2017.04.035 
27. Tavares, $D$, Almeida, R, Torres, DFM: Constrained fractional variational problems of variable order. IEEE/CAA J. Autom. Sin. 4(1), 80-88 (2017)

28. Abdelkawy, MA, Zaky, MA, Bhrawy, AH, et al.: Numerical simulation of time variable fractional order mobile-immobile advection-dispersion model. Rom. Rep. Phys. 67(3), 1-19 (2015)

29. Kumar, D, Singh, J, Baleanu, D: A new analysis for fractional model of regularized long-wave equation arising in ion acoustic plasma waves. Math. Methods Appl. Sci. (2017). doi:10.1002/mma.4414

30. Singh, J, Kumar, D, Qurashi, MA, et al.: A novel numerical approach for a nonlinear fractional dynamical model of interpersonal and romantic relationships. Entropy 19(7), 375 (2017). doi:10.3390/e19070375

31. Singh, J, Kumar, D, Swroop, R, et al.: An efficient computational approach for time-fractional Rosenau-Hyman equation. Neural Comput. Appl. (2017). doi:10.1007/s00521-017-2909-8

32. Lazo, MJ, Torres, DFM: Variational calculus with conformable fractional derivatives. IEEE/CAA J. Autom. Sin. 99, 1-13 (2016)

33. Jahanshahi, S, Torres, DFM: A simple accurate method for solving fractional variational and optimal control problems. J. Optim. Theory Appl. 174(1), 156-175 (2017)

34. Yang, XJ, Gao, F, Machado, JA, et al.: A new fractional derivative involving the normalized sinc function without singular kernel. (2017) arXiv:1701.05590

35. Atangana, A, Baleanu, D: New fractional derivatives with nonlocal and non-singular kernel: theory and application to heat transfer model. Therm. Sci. 20(2), 763-769 (2016)

36. Kilbas, AA, Srivastava, HM, Trujillo, JJ: Theory and Applications of Fractional Differential Equations. Elsevier, Amsterdam (2006)

37. Pishkoo, A, Darus, M: Fractional differintegral transformations of univalent Meijer's G-functions. J. Inequal. Appl. 2012(1), 1 (2012)

38. Kiryakova, $\vee$ : The special functions of fractional calculus as generalized fractional calculus operators of some basic functions. Comput. Math. Appl. 59(3), 1128-1141 (2010)

39. Algahtani, OJJ: Comparing the Atangana-Baleanu and Caputo-Fabrizio derivative with fractional order: Allen Cahn model. Chaos Solitons Fractals 89, 552-559 (2016)

40. Sheikh, NA, Ali, F, Saqib, M, et al:: A comparative study of Atangana-Baleanu and Caputo-Fabrizio fractional derivatives to the convective flow of a generalized Casson fluid. Eur. Phys. J. Plus 132(1), 54-68 (2017)

41. Koca, I, Atangana, A: Solutions of Cattaneo-Hristov model of elastic heat diffusion with Caputo-Fabrizio and Atangana-Baleanu fractional derivatives. Therm. Sci. 20(6), 2137-2147 (2016)

\section{Submit your manuscript to a SpringerOpen ${ }^{\circ}$ journal and benefit from:}

- Convenient online submission

- Rigorous peer review

Open access: articles freely available online

- High visibility within the field

- Retaining the copyright to your article

Submit your next manuscript at $\boldsymbol{~ s p r i n g e r o p e n . c o m ~}$ 